\title{
MOPSO using Probabilistic and Deterministic criteria based on OHL's Thermal Ratings
}

Link to publication record in Manchester Research Explorer

\section{Citation for published version (APA):}

Kapetanaki, A., \& Kopsidas, K. (2014). MOPSO using Probabilistic and Deterministic criteria based on OHL's Thermal Ratings. In 18th Power Systems Computation Conference (PSCC) IEEE.

\section{Published in:}

18th Power Systems Computation Conference (PSCC)

\section{Citing this paper}

Please note that where the full-text provided on Manchester Research Explorer is the Author Accepted Manuscript or Proof version this may differ from the final Published version. If citing, it is advised that you check and use the publisher's definitive version.

\section{General rights}

Copyright and moral rights for the publications made accessible in the Research Explorer are retained by the authors and/or other copyright owners and it is a condition of accessing publications that users recognise and abide by the legal requirements associated with these rights.

\section{Takedown policy}

If you believe that this document breaches copyright please refer to the University of Manchester's Takedown Procedures [http://man.ac.uk/04Y6Bo] or contact uml.scholarlycommunications@manchester.ac.uk providing relevant details, so we can investigate your claim.

\section{OPEN ACCESS}




\section{MOPSO using Probabilistic and Deterministic criteria based on OHL's Thermal Ratings}

\author{
Alexandra Kapetanaki \\ Electrical Energy and Power Systems \\ University of Manchester \\ Manchester, UK \\ alexandra.kapetanaki@manchester.ac.uk
}

\author{
Konstantinos Kopsidas \\ Electrical Energy and Power Systems \\ University of Manchester \\ Manchester, UK \\ k.kopsidas@manchester.ac.uk
}

\begin{abstract}
A Population Intelligent (PI) methodology called Particle Swarm Optimization has recently been applied to power system networks with the view to minimize the computational burden of Monte Carlo Simulation in the reliability domain. This paper presents a novel Multi Objective Particle Swarm optimization (MOPSO) methodology which adapts traditional binary PSO to multi objective PSO and intelligently prunes the state space by using the thermal capacity of transmission lines derived from the more detailed modelling of OHLs. For the implementation of the algorithm, deterministic metrics are used to evaluate the loading of the lines with the view to further enhance the efficiency of the proposed method. The IEEE 24-bus RTS is used under different case studies to validate that the filtering based methodology achieves computational effectiveness as well as improves network performance indices.
\end{abstract}

Keywords - adequacy; multi objective optimization technique; thermal rating; deterministic and probabilistic studies; network security

\section{Introduction}

Network operators usually implement deterministic N-1 (or $\mathrm{N}-2$ and/or N-D) outage criteria [1]. Through the implementation of any of these criteria, operators inherently rely on the pre-supposition that the network will not be exposed to the risk of load curtailment following 1 or 2 (or double circuit) component losses. Nonetheless, this assumption is flawed, because in reality the system is always exposed to risk of failure; and subsequently to customer outages in spite of the operator's ability to minimize this risk by implementing post fault corrective actions. Given these reasons, probabilistic methods have been proposed to tackle these problems [2]-[5].

In principle, the reliability criterion under probabilistic quantification is superior to the one relinquished through deterministic quantification. Therefore, there is an imperative need to adopt probabilistic metrics upon which to generate solutions that guarantee to optimize and enhance network operation and planning [6]. The metrics of utilising a probabilistic framework are rendered through their inherent ability to account for the vast range of uncertainties that surround the behaviour of generation and transmission units. Thus historically, by simulating the actual processes of system based random behaviour through techniques such as the Monte Carlo Simulation (MCS) reliability engineers have been able to accurately measure system performance through the estimation of probabilistic indices of interest [7]. However, its inherent ability to account for the uncertainties of the generation, transmission, demand makes it to be computationally infeasible. Simulation techniques such as MCS usually used to estimate the probabilistic indices by simulating the actual process and random behaviour of the system [7]. Due to its large computational burden in recent years, many efforts have been made to enhance MCS in order to improve its computational efficiency. Such efforts are pseudochronological MCS [8],[9], Cross Entropy (CE) [10], [11], Quasi-sequential, Quasi-CE [12] and Latin Hypercube Sampling (LHS) [13], which are combined with conventional MCS with the view to accelerate its CPU time. The last five years Population Intelligent Search (PIS) have been applied to the probabilistic reliability analysis of power systems. The synergistic combination of those and MCS have resulted in intelligent optimization techniques, which improve the method of sampling the traditional MCS. These applications have mainly been focused on the use of GA [14], [15] and PSO [16], but there are also some works including AIS and ACO. Such works are [17] and [18], where in the former investigations regarding the combination of PIS Methods (GA, PSO, ACO and AIS) and composite system reliability evaluation incorporating wind farm integration are examined and in the latter BPSO, GA and BACO techniques demonstrate computational robust, since they dramatically decrease the CPU time as well as the MCS iterations that are required for convergence in both IEEE RTS 79 and IEEE RTS 96 networks. Nonetheless, much attention has been placed on PSO, since it has been proved the most versatile method due to its ability to solve multi objective problems and so it can accommodate the new features that a smart grid poses to electrical power networks.

In this context, this paper focuses on extending the single objective PSO to MOPSO state space pruning methodology that can be a significant premise in accomplishing reliability evaluation of power complex systems incorporating the thermal ratings of transmission lines. In particular, the proposed MOPSO or filtering technique is developed subject to three objective functions:1) probability of a given state, 2) total load curtailment in a given state, 3) transmission system capacity considering weight indexes, which distinguish the importance of the lines. MOPSO and MCS modelling methods will further enhance the reliability evaluation of transmission operation using more detailed OHL properties. 


\section{RELIABILITY ASSESMENT MODELLING WITH MOPSO}

\section{A. Particle Swarm Optimization technique}

The Particle Swarm Optimizer (PSO) is a population based optimization technique first proposed by Kennedy and Eberhart [19]. PSO, similarly to the algorithms belonging to the Evolutionary Algorithm family, is a stochastic algorithm that can be used on functions where the gradient is either unavailable or computationally expensive to obtain. The origins of PSO are best described as sociologically inspired, since the original algorithm was based on the sociological behaviour associated with bird flocking and school of fish [20]. The algorithm maintains a population of particles, where each particle represents a potential solution to an optimization problem. If the size of the swarm is for example s, each particle i can be represented as an object with several characteristics. These characteristics are assigned the following symbols:

$\mathbf{x}_{\mathbf{i}}:$ the current position of the particle;

$\mathbf{v}_{\mathbf{i}}$ : the current velocity of the particle

$\mathbf{y}_{\mathbf{i}}$ : the personal best position of the particle

The personal best position associated with particle $\mathrm{i}$ is the best position that the particle has visited (a previous value of $\mathrm{x}_{\mathrm{i}}$ ), yielding the highest fitness value for that particle. Each particle characterized by a gbest value, which denotes the best position discovered by any of the particles so far. The algorithm makes use of two independent random sequences, $r_{1}$ $\sim \mathrm{U}(0,1)$ and $\mathrm{r}_{2} \sim \mathrm{U}(0,1)$ which are combined with the particle's personal and global best solution to update the velocity and the position of each particle.

$$
\begin{aligned}
v_{i, j}(t+1)=v_{i, j}(t) & +F_{1} r_{1, j}(t)\left[\operatorname{PPr} \operatorname{Pest}_{i, j}(t)-x_{i, j}(t)\right] \\
+ & V_{1} r_{2, j}(t)\left[\operatorname{Gpbest}(t)-x_{i, j}(t)\right] \\
+ & \ldots \\
x_{i}(t+1) & =x_{i}(t)+v_{i}(t+1)
\end{aligned}
$$

The velocity update step is specified separately for each dimension $j=1 \ldots . n$, so that $v_{i, j}$ denotes the $j^{\text {th }}$ dimension of the velocity vector associated with the $\mathrm{i}^{\text {th }}$ particle.

A binary version of the PSO was introduced by Kennedy and Eberhart [21]. This alternative of PSO restricts the component values of $x_{i}$ and $y_{i}$ to be elements taken from the sets $(0,1)$. The velocities can be calculated by introducing a logistic sigmoid transformation function, see (1.3) and positions are updated through (1.4):

$$
\begin{gathered}
\operatorname{sig}\left(v_{i, j}(t+1)\right)=\frac{1}{1+\exp \left(-v_{i, j}(t+1)\right)} \\
x_{i, j}(t+1)= \begin{cases}0 & \text { if } \operatorname{rand}()_{j}(t) \geq \operatorname{sig}\left(v_{i, j}(t+1)\right) \\
1 & \text { if } \operatorname{rand}()_{j}(t)<\operatorname{sig}\left(v_{i, j}(t+1)\right)\end{cases}
\end{gathered}
$$

In the reliability domain single Binary PSO (BPSO) uses one objective function to identify the loss of load states and evaluates the performance of reliability indexes [16], [17], [22].

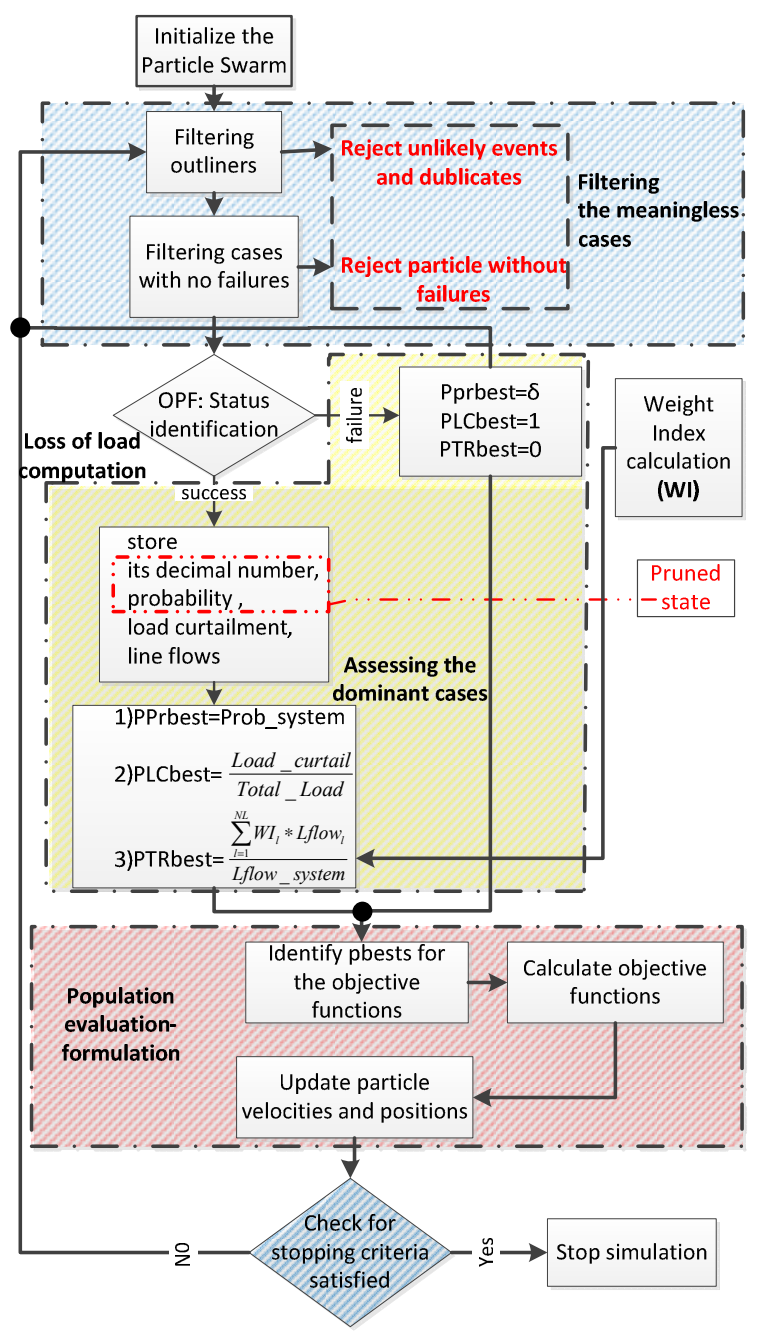

Fig. 1. MOPSO flowchart.

Correspondingly, Multi objective PSO uses multiple fitness functions to prune the state space [23]-[25].The velocities are calculated by (1.1) and the particles' positions by (1.4).

\section{B. The proposed MOPSO algorithm}

The main goal of the proposed algorithm is to define faster the states which contribute less to the computation of the reliability indexes. These states are characterized as success states or non-loss of load states and have high probability. Towards this, a success status can be obtained either by evaluating the power flow of the lines (normal or alert state) or the load curtailment. Therefore, the movement of the particle is accomplished in a 3 dimension space searching for the optimal solution subject to three coordinates. The first objective function is based on the maximization of the probability of the system state. As such, the success states occur with higher density compared to failure states. The second objective function uses the minimization of load curtailment to further encourage the particle's movement to a success state and the third objective function makes use of the maximization of the rating of the transmission lines with the view to direct the particle faster to the most frequently success state by MCS. This function is explained as follows: 


$$
\begin{aligned}
& v_{i, j}(t+1)=v_{i, j}(t)+F_{1} r_{1, j}(t)\left[\text { PPr } \text { best }_{i, j}(t)-x_{i, j}(t)\right] \\
& +V_{1} r_{2, j}(t)\left[\text { Gpbest }(t)-x_{i, j}(t)\right] \\
& +F_{2} r_{3, j}(t)\left[\operatorname{PLCbest}_{i, j}(t)-x_{i, j}(t)\right] \\
& +V_{2} r_{4, j}(t)\left[\operatorname{Glcbest}(t)-x_{i, j}(t)\right] \\
& +F_{3} r_{5, j}(t)\left[\text { PTRbest } t_{i, j}(t)-x_{i, j}(t)\right] \\
& \quad+V_{3} r_{6, j}(t)\left[\operatorname{Gtrbest}(t)-x_{i, j}(t)\right]
\end{aligned}
$$

PPrbest $\mathrm{t}_{\mathrm{ji}}$ addresses the highest system state probability;

Gpbest denotes the best position with the highest system state probability discovered by any of the particles so far;

PLCbest $t_{i}$ addresses the minimum load curtailment; Glcbest denotes the best position with the lowest load curtailment discovered by any of the particles so far PTRbest $\mathrm{t}_{\mathrm{ji}}$ addresses the highest thermal rating; Gtrbest denotes the best position with the highest rating discovered by any of the particles so far

- MOPSO algorithm

The steps of the proposed methodology are illustrated in Fig.1:

Initialization: The number of the particles $\mathrm{P}$ and generations $\mathrm{G}$ are set to the simulation. The components of the system (generators and lines) are represented using a binary encoding scheme; 1 represents the up and 0 the down status of the dimensions of each particle. For each dimension $\mathrm{j}$ of a particle the initial positions and velocities are defined by the Forced Outage Rate (FOR) of the $\mathrm{j}^{\text {th }}$ dimension (total number of generators and lines), as follows.

$$
\begin{aligned}
& X_{i, j}=\left\{\begin{array}{ll}
0 & r \leq F^{\prime} R_{i, j} \\
1 & \text { otherwise }
\end{array}\right\} \\
& v_{i, j}=\left\{\begin{array}{cc}
F O R_{i, j} & X_{i, j}=0 \\
1-F O R_{i, j} & X_{i, j}=1
\end{array}\right\}
\end{aligned}
$$

$X_{i, j}$ is the value of the position of the $j^{\text {th }}$ dimension of the $i^{\text {th }}$ particle

$\mathrm{v}_{\mathrm{i}, \mathrm{i}}$ is the value of velocity vector

Since all the particles obtain a position vector at position $x_{i}$ and velocity vector $\mathrm{V}_{\mathrm{i}}$, they track a personal best solution and position that relates to the multi-function.

Filtering meaningless cases: The network status is considered as failure state due to the following cases: a) the probability of the system state is lower than a very small number $\delta ; b$ ) a particle is the same with a particle of the same or previous generation; c) all the components are designated in the up state

Loss of load computation: DC OPF uses as objective the minimization of total load curtailment to determine the loss of load states as fail states and the reverse. The objective function is derived from the following equations and it uses piecewise linear approach for the minimization of loss of load. Therefore, the minimization of loss of load and generation cost are optimized with linear approach and not with the proposed heuristic algorithm.

$$
\min \sum_{i=1}^{N_{b}} C_{i}
$$

Subject to

$B_{b u s} \theta-P_{g}-C=-P_{d}$

$B_{f} \theta \leq P_{\text {line }}^{\max }$

$-B_{f} \theta \leq P_{\text {line }}^{\max }$

$0 \leq C \leq P_{d}$

$P_{\min } \leq P_{g} \leq P_{g}^{\max }$

$\mathrm{Ci}$ is the load curtailment vector at each bus i, Bbus is a susceptance matrix, $\mathrm{Bf}$ is a susceptance matrix at from bus, $\theta$ is a voltage angle level, $\mathrm{Pg}$ is a generator real power injection; Pd is real power demand; $P_{\text {line }}^{\max }, P_{\text {line }}^{\min }$ are transmission line limits, $\mathrm{Nb}$ is the number of buses of the IEEE RTS system.

Weight index calculation: the novelty of this paper is the use of a new index to weight the loading of the lines. Deterministic (N-1) approach is implemented for the calculation of the index. After sensitivity analysis was implemented, the occurrences that a line is loaded above given ratings were recorded for the peak load. The analysis was implemented considering $\mathrm{N}-1$ outages of the lines, therefore for all system states the Probability equals to 1 . The system is operated under the occurrence of a credible outage without causing voltage instabilities and load shedding. The Probability (Pi) of each state is calculated by using (1.11), assuming that all outages are independent. The threshold of overloadings set to have values from $10 \%$ to $100 \%$ of the actual ratings of the lines with increments of 10 . Therefore, the frequency $\left(\mathrm{WI}_{\mathrm{D}}\right)$ of OHLs' overloadings was determined for the various rating limits (1.10). The average value of $\mathrm{WI}_{\mathrm{D}}$ for each OHL forms the WI equation (1.9) aiming to show the importance of each line in the network:

$$
\begin{aligned}
& W I=\frac{\sum_{i^{*} 10}^{N} W I_{D, i}}{N} \\
& W I_{D}=\frac{\sum_{s \in \Omega(t)} \text { Pov }_{s}}{\text { Simulation_year }_{-}} \\
& P_{i}=\left\{\begin{array}{cc}
1-F O R_{i} & \text { up } \\
F O R_{i} & \text { down }
\end{array}\right. \\
& F O R=\frac{\lambda}{\lambda+\mu}
\end{aligned}
$$$$
W I_{D} \text { : }
$$

$\begin{array}{ll}\text { Pov }_{s}: & \text { deterministic approach }(\mathrm{i}=1, \ldots, 10) \\ & \text { Overloaded probability of OHLs of system } \\ & \text { state } \mathrm{s} \\ \mu: & \text { failure rate of the units } \\ \mathrm{N}=10 & \text { repair rate of the units }\end{array}$

Assessing the dominant cases: If the particle is a success state then the algorithm processes the following steps a) store the 
binary sequence of the particle as pruned state; b) store the probability; c) store the load curtailment of the system state and store the power flows of the transmission lines. Otherwise, set to the Pbests low importance values and go to the next particle.

Population evaluation-formulation: Determine the Pbests by using the inputs of the network status. Update the velocities and positions for each dimension $\mathrm{j}$ of particle $\mathrm{i}$ using (1.5) and check the following constraints:

If $\left.v_{i, j}^{k+1}\right\rangle V_{\max }$ then $v_{i, j}^{k+1}=V_{\max }$

If $v_{i, j}^{k+1}\left\langle V_{\min }\right.$ then $v_{i, j}^{k+1}=V_{\text {min }}$

$\mathrm{V}_{\max }$ and $\mathrm{V}_{\min }$ are considered as a threshold for the velocityprobability of failure and make use different values for the lines and for the generators in order to make the algorithm more realistic.

Stopping Criteria: The simulation process is continued until the number of the particle generations is reached $\left(\mathrm{k}=\mathrm{G}_{\max }\right)$.

\section{Monte Carlo Simulation}

MOPSO is combined with Monte Carlo Simulation in order to discard a great amount of success state and make MCS converge more quickly. The MC algorithm used for this purpose can be divided into the following steps:

- Set the number of samples

- Use state sampling technique to determine the system state. For the sampling technique the random numbers are taken exponentially from the range $(0,1)$.

- If the state has been pruned determine from the database whether it is a success or failure state. If it is a success state discard this event. If not add the state into the calculation of LOLP.

- If the state has not been pruned run DC OPF and classify it as a success or failure state.

- If it is a failure state then add the state into the calculation of LOLP using the following equations

$$
L O L P_{M O P S O-M C S}=L O L P_{M C S} *\left(1-\sum P_{i}\right)
$$

$\mathrm{P}_{\mathrm{i}}$ is the probability of occurrence of a pruned state generated by the PSO.

$$
\begin{gathered}
L O L P_{M C S}=\frac{1}{K} \sum_{i=1}^{K} D_{i} \\
D_{i}=\left\{\begin{array}{lc}
1 & \text { loss of load state } \\
0 & \text { othwerwise }
\end{array}\right.
\end{gathered}
$$

- If the stopping criterion is fulfilled $(\sigma \leq 0.04)$, then stop simulation[7].

The index used in this paper to evaluate the reliability of implementing STR, SeTR and TVTR is the energy expected not supplied (EENS, MWh/y) and is given by (1.15)

$$
\text { EENS }=\sum_{i=1}^{N L}\left(\sum_{s e F_{i}} P(s) C(s)\right) T_{i}
$$

$\mathrm{C}(\mathrm{s})$ is the load curtailment in state $\mathrm{s}$

$\mathrm{P}(\mathrm{s})$ is the probability of system state $\mathrm{s}$

NL is the number of load levels

$\mathrm{T}_{\mathrm{i}}$ is the total number of the one period cycle (hours)

$F_{i}$ is the set of all system states associated with load curtailment

\section{CASE STUDIES: ILLUSTRATION, VALIDATION AND ANALYSIS ON A 24-NODE NETWORK}

In this section the proposed MOPSO is applied to the IEEE RTS79 [26]. The IEEE system contains 38 lines and 32 generators of varying types such as hydro, coal/steam, nuclear. The total peak load of the system is $2850 \mathrm{MW}$, while the total generating capacity is $3405 \mathrm{MW}$. In order for the proposed model to demonstrate its robustness, only the peak load levels were used for the analysis as well as elevated FOR for the transmission lines.

The software was written in Matlab language using modified Matpower for the power flow calculations[27]. Matpower and more specifically Gurobi are used to solve the DC OPF with minimization of the load curtailment and minimization of the generation costs objective functions. The simulations were run in a computer with Intel Core i7-3820 CPU processor and 32 GB RAM.

\section{A. MOPSO-Results}

The method described in section II.B was implemented using acceleration factors: $\mathrm{F} 1=0.01, \mathrm{~V} 1=0.1 ; \mathrm{F} 2=0.1, \mathrm{~V} 2=0.1$; $\mathrm{F} 3=0.1, \mathrm{~V} 3=0.1$ and velocity limit $\mathrm{V}_{\max }=4$ and $\mathrm{V}_{\min }=-4$. The size of the swarm also plays a determining role in the convergence of NMCS. Due to this, each simulation run for a combination of particles and generations, which range from 5 to 30 particles and from 50 to 300 generations. After the various combinations of particles and generations completed the data was sorted in ascending order in respect to the number of success states pruned. The probability threshold of system states is $\delta=10^{-7}$ and the stopping criterion for NSMCS is $4 \%$. NMCS is used as a baseline to calculate the LOLP and computational time of the crude method. Both the effects of transmission lines and generators' failures are considered on reliability models.

Three measurements of performance were computed: LOLP, $\mathrm{CPU}$ and number of iterations. Table I presents comparisons of system indices between NSMCS (Baseline) and the proposed MOPSO.

TABLE I.

COMPARISON OF SYSTEM INDICES USING NSMCS, MOPSO

\begin{tabular}{lcc}
\hline \hline $\begin{array}{c}\text { RELIABILITY } \\
\text { INDICES }\end{array}$ & NSMCS & MOPSO \\
\hline LOLP & 0.06261 & 0.06359 \\
\hline iterations & 85534 & 24625 \\
\hline CPU(hr) & 3.23 & 0.8614 \\
\hline
\end{tabular}

Fig. 2 detail the comparative results between single, bi, multi based objective functions for pruning the state. In particular it indicates that the LOLP derived from the optimization algorithm shows high accuracy in the range of 0.004 in respect to the baseline LOLP. In addition, the proposed MOPSO displays slightly smaller LOLP fluctuation around the baseline throughout the total number of trials, which 


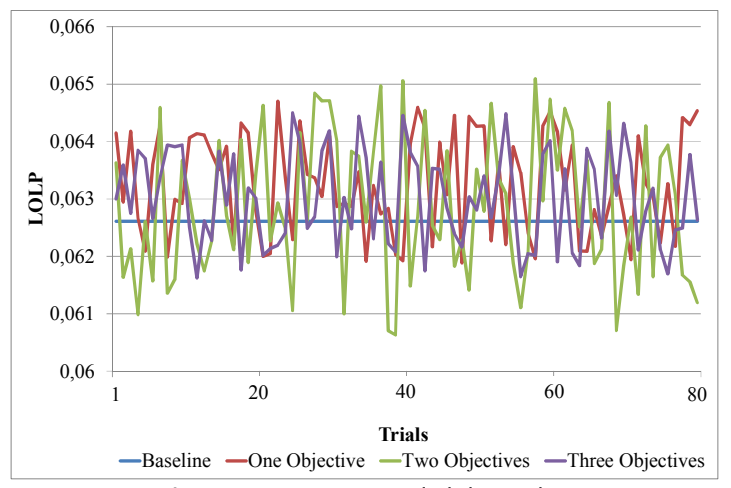

Fig. 2. MOPSO: LOLP reliability index

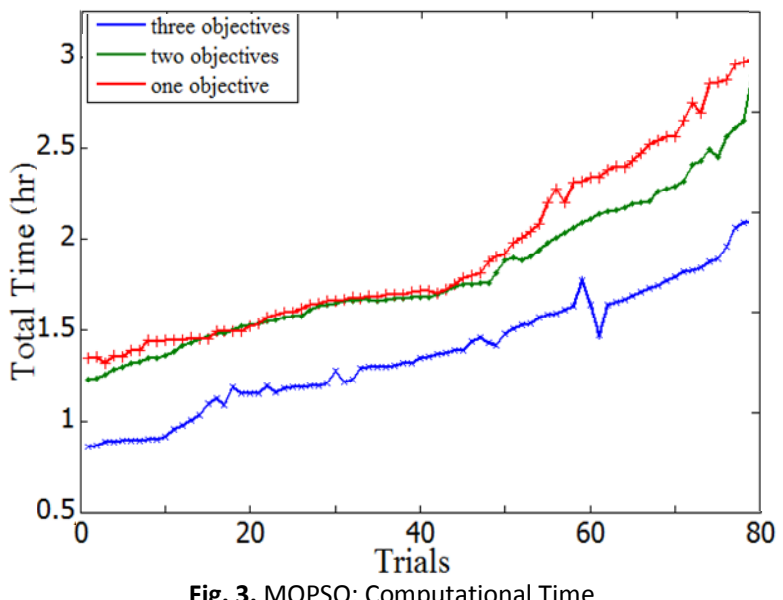

Fig. 3. MOPSO: Computational Time

demonstrates that the novel method improves network performance indices.

Fig. 3 shows the total computational time required from both MOPSO to complete and MCS to converge. It is clear that the proposed MOPSO is faster than the single and bi-objective function. In particular, MCS needs $1.35 \mathrm{hr}$ when the single objective function is used for pruning, while the proposed method (three-objectives) decreases the MC convergence to about $0.85 \mathrm{hr}$. This is because the MOPSO prunes larger number of success states for the same combination of particles and generations due to it combines the finer aspects of the three objectives and so being superior to either objective on its own. The spikes that occur in all three optimization algorithms mean that although the number of success states pruned is large, the number of MCS iterations is bigger due to there is a possibility for MCS to randomly sample a success system state that it wasn't captured by the optimization techniques (collision). Furthermore, it can be inferred from the graph that the computational time of PSO subject to one objective function is almost the same between 20 and 40 trials. This is mainly due to the bi-objective function identifies success states which are not sampled so often by MCS as well as due to the occurrence of collisions between PSO method and MCS. In summary from the graph it is indicated a stark difference of $74 \%$ in CPU comparing the proposed MPSO $\left(1^{\text {st }}\right.$ trial $)$ with the CPU time of the MCS algorithm alone given in Table I. Fig. 4 demonstrates that the MOPSO method prunes a number of success states that is clearly more than both the probability based method and the probability-curtailment method. All methods prune up to 14500

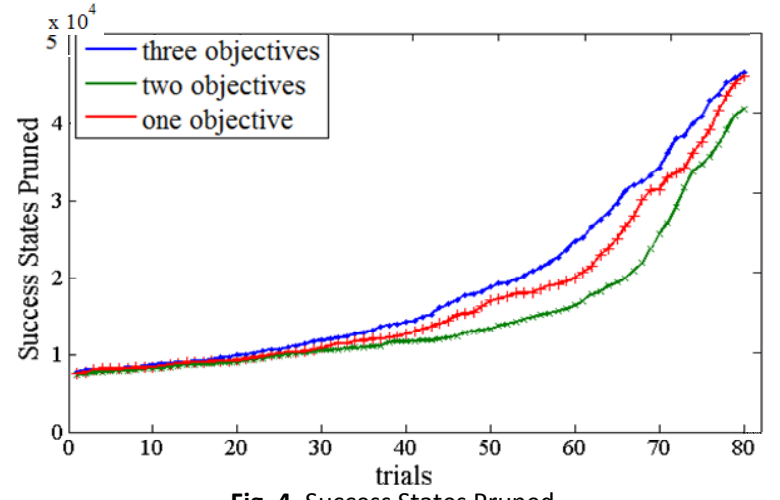

Fig. 4. Success States Pruned

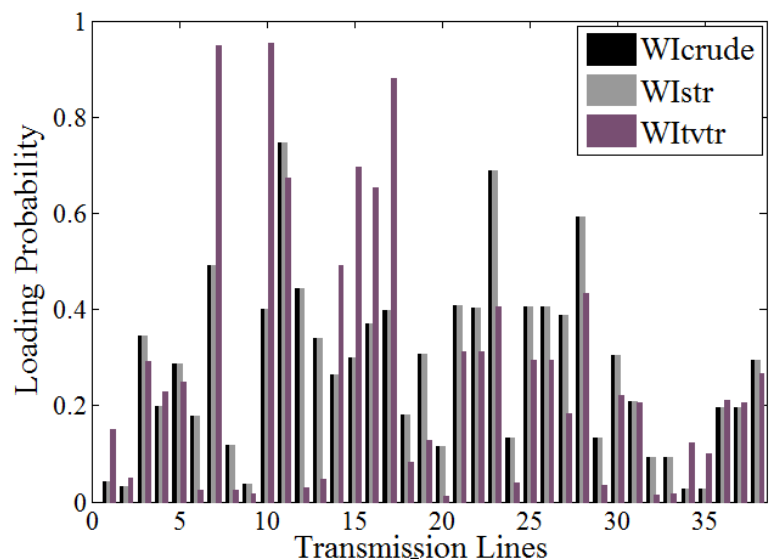

Fig. 5. Weight Indexes (WI) used by the proposed MOPSO for the various thermal rating scenarios

success states for the first 40 simulations and then the pruning increase in a sigmoid nature.

It can be seen that the algorithm using one objective function, it prunes more success states than the method with two objective functions. This is evident, since the algorithm with two objective functions is mainly affected by the curtailment objective function, which plays determining role in the selection of the success state. However, PSO using two objectives shows better results, see Fig.3, because the occurrence of success states pruned by two objectives are more frequently compared to one objective function.

The Weight Index ( $\mathrm{WI}_{\text {crude }}$ ) used to validate the proposed MOPSO uses the capacity data of the transmission lines given by the IEEE RTS. The loading of each transmission line is computed after deterministic analysis implementation using the peak load of IEEE RTS for the $\mathrm{WI}_{\text {crude }}$ and 2.5 times the peak load of the network for the $\mathrm{WI}_{\text {str }}$ (static thermal rating) and $\mathrm{WI}_{\text {tvtr }}$ (time varying thermal rating), which are introduced in the next section (II B). It is illustrated that for the $138 \mathrm{kV}$ voltage IEEE RTS network line 11 is the most frequently overloaded, whereas for the $230 \mathrm{kV}$ network lines 23 and 28 show extremely high loading probability. Therefore, it can be implied that the indexes of the most critical lines boost the intelligent movement of particles to search and track more success system states. This is due to the ability of particle to discover the states that the lines are overloaded subject to generator and line failures in order to satisfy the total demand and eventually result in success state. 


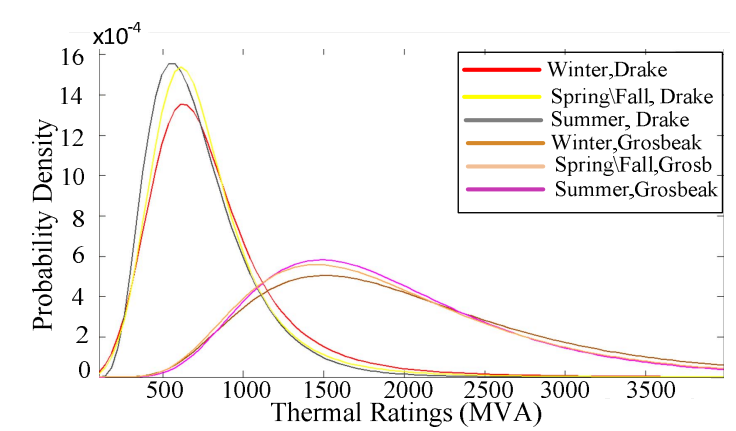

Fig. 6. Probability Distribution Functions of SeTR of Aonach UK area for two different conductors

\section{B. Application of MOPSO method considering OHL's properties}

A study was undertaken on a modified IEEE 24 bus network. The system was divided into two Voltage systems: the $138 \mathrm{kV}$ network and the $230 \mathrm{kV}$ network, which both use ACSR conductors, single bundle drake and twin bundle grosbeak conductor respectively. The electrical properties of these conductors as well as the weather data of the geographical area the conductor is located in are used in order for the thermal capacity of the line to be calculated. The conductor characteristics are defined by the diameter, the calculated resistance at $25^{\circ} \mathrm{C}$ and $75^{\circ} \mathrm{C}$, as well as its reactance provided by the IEEE RTS. The weather data consists of wind speed $\left(\mathrm{V}_{\mathrm{m}}\right)$, direction $\left(\mathrm{K}_{\text {angle }}\right)$, and ambient temperature $\left(\mathrm{T}_{\mathrm{a}}\right)$. Finally the load parameters constitute the ampacity of the lines for a given load level. The meteorological data of 5 years from 1997 to 2001 were obtained from BADC Met office MIDAS stations for Aonach UK area [28]. According to the deterministic approach included in IEEE standard [29] the maximum current that a line can carry can be derived by the steady state balance heat equation as shown in (1.16)

$$
\begin{aligned}
& I= \sqrt{\left(P_{c}\left(T_{c}, T_{a}, K_{\text {angle }}, V_{m}\right)+P_{r}\left(T_{a}, T_{c}\right)-P_{s}\right) / R\left(T_{c}\right)} \\
& \mathrm{P}_{\mathrm{c}}=\text { convection heat loss, } \mathrm{W} / \mathrm{m} \\
& \mathrm{P}_{\mathrm{r}}=\text { radiated heat loss, } \mathrm{W} / \mathrm{m} \\
& \mathrm{P}_{\mathrm{s}}=\text { solar heat gain, } \mathrm{W} / \mathrm{m} \\
& \mathrm{R}=\text { ac conductor resistance at operating temperature, } \Omega / \mathrm{m} \\
& \mathrm{I}=\text { conductor current, amperes } \\
& \mathrm{T}_{\mathrm{c}}=\text { conductor temperature, }{ }^{\circ} \mathrm{C}
\end{aligned}
$$

For simplicity, the solar heat gain parameter is omitted in this work since the effect of solar irradiation on the conductor rating is small. The proposed model tested considering Static (STR), Seasonal Thermal Rating (SeTR) and Time Varying Thermal Rating (TVTR) with the view to test how the third objective function affects the pruning of the state space.

1) STR: The STR is determined based on the following parameters: maximum ambient temperature $T_{a}$ in Aonach area historically, which equals to $20^{\circ} \mathrm{C}$, wind speed to $0.61 \mathrm{~m} / \mathrm{sec}$, $\mathrm{K}_{\text {angle }}$ to 1 and $\mathrm{T}_{\mathrm{c}}=95^{\circ} \mathrm{C}$ (maximum elevated temperature for ACSR for ACSR conductors).

2) SeTR: Similar to the STR, the fixed thermal rating is usually calculated for each season. Three different ratings are considered: 1) for summer, 2) for spring/ fall and 3) for winter. To do so, for every conductor the hourly weather information

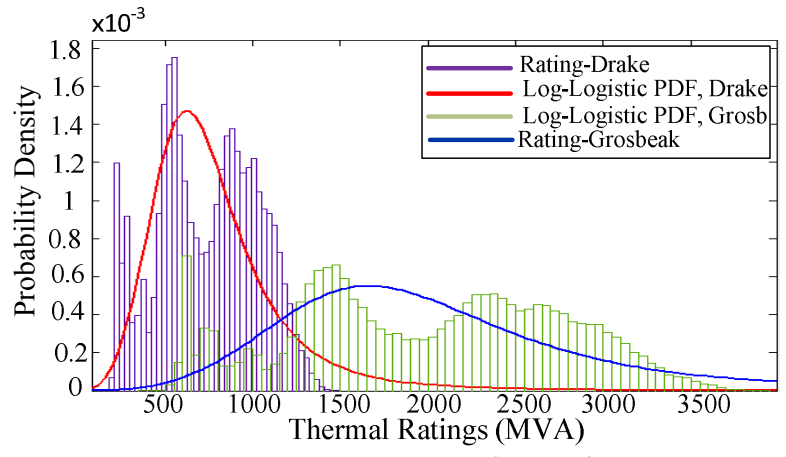

Fig. 7. Probability Distribution Functions of TVTR of Aonach UK area for two different conductors

as well as the parameters of conductor are plugged into (1.16) to calculate five years hourly thermal rating for every season. The probability distribution function (PDF) of SeTR for Aonach area are shown in Fig. 6. The median of each season is used to test the proposed MOPSO. Since both NSMC and MOPSO are not time dependent, at each sample of the system state the season is selected randomly. In particular, for winter the random number is designated between 1-1416 hours and 8017-8760 hours, for summer between 3625-5832 hours anf for spring/fall between 1417 and 3624 hours.

3) TVTR: In order to determine the real transmission capacity that is available on a given network, the hourly weather information as well as the parameters of the conductor are plugged into (1.16) to calculate 5 years hourly thermal rating. The variations of thermal ratings are modelled with a PDF. Among all standard distributions, the best distribution function matching with calculated hourly thermal ratings is the log-logistic, see Fig. 7 which is formulated by (1.17) .

$$
\begin{aligned}
& f(x / \mu, \sigma)=\frac{1}{\sigma} \frac{1}{x} \frac{e^{z}}{\left(1+e^{z}\right)^{2}} \\
& z=\frac{\log (x)-\mu}{\sigma}
\end{aligned}
$$

The PDFs generate the maximum TR at each sample of system state for both MCS and MOPSO. Subsequently, the load flow (Lflow) parameters of the lines are calculated after using DC (OPF) formulation for the network considering the operating conditions (i.e. failures if any) and the initial maximum thermal rating. The TRs are set on the MOPSO algorithm as it is shown in the flowchart of Fig.1 through the "assessing the dominant cases" step. It should be stressed that in the analysis the $T_{c}$ is set at $95^{\circ} \mathrm{C}$ based on the avoidance of the conductor annealing [30][31].

The additional scenario with increased load to $2.5 \mathrm{pu}$ its peak load is also used taking into consideration the thermal ratings of the OHLs. Table II presents the reliability indices and CPU for the various thermal ratings scenarios. It is demonstrated that TVTR facilitates MOPSO algorithm. This is more likely because the more detailed parameters of OHLs enhance the efficiency of the algorithm as well as the various thermal ratings values enable the proposed algorithm to escape from local minima and maxima. In particular, $\mathrm{MOPSO}_{\mathrm{TVTR}}$ is $73.5 \%$ faster than $\mathrm{NSMCS}_{\mathrm{TVTR}}$, whereas $\mathrm{MOPSO}_{\mathrm{STR}}$ is $67.2 \%$ faster than $\mathrm{NSMCS}_{\mathrm{STR}}$. At the same time MOPSO calculates accurate the LOLP and EENS indexes. In addition, the TVTR model resulted in $46.54 \%$ lower EENS than the STR model. 
This is mainly due to the increased capacity of transmission lines provided from the TVTR model.

TABLE II.

\begin{tabular}{|c|c|c|c|c|}
\hline $\begin{array}{l}\text { RELIABILITY } \\
\text { INDICES }\end{array}$ & LOLP & $\begin{array}{c}E E N S \\
(M W h r / y)\end{array}$ & iterations & $\begin{array}{l}\mathrm{CPU} \\
(\mathrm{hr})\end{array}$ \\
\hline $\mathrm{NSMCS}_{\mathrm{STR}}$ & 0.3898 & 28.25 & 14146 & 0.55 \\
\hline $\mathrm{NSMCS}_{\mathrm{SeTR}}$ & 0.3574 & 21.01 & 32983 & 0.98 \\
\hline $\mathrm{NSMCS}_{\mathrm{TVTR}}$ & 0.3429 & 15.1 & 59402 & 1.62 \\
\hline MOPSO $_{\text {STR }}$ & 0.3885 & 28.13 & 3879 & 0.18 \\
\hline MOPSO $_{\text {SeTR }}$ & 0.3591 & 20.87 & 7916 & 0.27 \\
\hline MOPSO $_{\text {TVTR }}$ & 0.3418 & 15.1 & 10710 & 0.43 \\
\hline
\end{tabular}

\section{CONCLUSION \& FURTHER WORK}

This paper presents the newly developed method based on multi-objective PSO for reliability assessment using three objective functions. The method has shown significant advantage regarding both the performance indices and the reduction of the simulation time of NSMCS. Furtheremore, thermal ratings (STR, SeTR, TVTR) of transmission lines using OHL's properties is used to assess the effectiveness of the proposed model. It is highlighted that the computational effort required by the proposed MOPSO algorithm using TVTR is only $26.5 \%$ compared to the MCS (baseline).

Further work should be done to show the efficiency of this algorithm including time varying thermal ratings in both IEEE79 and to a larger system, such as IEEE RTS96 system considering multiple load levels.

\section{REFERENCES}

[1] L. Söder, J. Douglas, and A. Ekwue, "Study on the Technical Security Rules of the European Electricity Network," Stockholm, 2006.

[2] W. Li and P. Choudhury, "Probabilistic Transmission Planning," IEEE Power Energy Mag., vol. 5, pp. 46 - 53, 2007.

[3] J. D. McCalley et al., "Probabilistic security assessment for power system operations," in Power Engineering Society General Meeting, 2004 , pp. $212-220$.

[4] J. M. Arroyo and N. Alguacil, "A Risk-Based Approach for Transmission Network," IEEE Trans. Power Syst., vol. 25, no. 3 , pp. 1759-1766, 1995.

[5] D. S. Kirschen, K. R. W. Bell, D. P. Nedic, D. Jayaweera, and R. N. Allan, "Computing the value of security," in IEE ProceedingsGeneration, Transmission and Distribution, 2003, vol. 150, pp. 673 $-678$.

[6] R. Moreno, D. Pudjianto, and G. Strbac, "Transmission Network Investment with Probabilistic Security and Corrective Control," IEEE Trans. POWER Syst., vol. 28, no. 4, pp. 3935-3944, 2013.

[7] R. Billinton and W. Li, Reliability Assessment of Electrical Power Systems Using Monte Carlo Methods. New York: London:Plenum, 1994, pp. 35-36.

[8] A. M. Leite, A. Luiz, F. Manso, J. C. de O. Mello, and R. Billinton, "Pseudo-Chronological Simulation for Composite Reliability Analysis with Time Varying Loads," IEEE Trans. POWER Syst., vol. 15 , no. 1,2000

[9] J. C. O. Mello, M. V. F. Pereira, and A. M. L. Da Silva, "Evaluation of reliability worth in composite systems based on pseudosequential Monte Carlo Simulation," IEEE Trans. Power Syst., vol. 9, no. 3, 1994.

[10] A. Silva, A. G. Fernández, and C. Singh, "Generating Capacity Reliability Evaluation Based on Monte Carlo Simulation and CrossEntropy Methods," IEEE Trans. Power Syst., vol. 25, no. 1, pp. 129-137, 2010.

[11] D. Ernst, M. Glavic, G.-B. Stan, S. Mannor, and L. Wehenkel, "The cross-entropy method for power system combinatorial optimization problems," in IEEE Lausanne Power Tech, 2007, pp. 1290-1295.
[12] R. A. González-Fernández and A. M. Leite da Silva, "Reliability Assessment of Time-Dependent Systems via Sequential CrossEntropy Monte Carlo Simulation," IEEE Trans. Power Syst., vol. 26, no. 4, pp. 2381-2389, 2011.

[13] Z. Shu and P. Jirutitijaroen, "Latin Hypercube Sampling Techniques for Power Systems Reliability Analysis With Renewable Energy Sources," IEEE Trans. Power Syst., vol. 26, no. 4, pp. 2066-2073, 2011.

[14] R. Green, L. Wang, and C. Singh, "State Space Pruning for Power System Reliability Evaluation using Genetic Algorithms," IEEE Power Energy Soc. Gen. Meet., pp. 1-6, 2010.

[15] R. Green, Z. Wang, L. Wang, M. Alam, and C. Singh, "Evaluation of Loss of Load Probability for Power Systems Using Intelligent Search Based State Space Pruning," in IEEE 11th International Conference on Probabilistic Methods Applied to Power Systems (PMAPS), 2010, pp. 319 - 324.

[16] R. Green, L. Wang, and C. Singh, "State Space Pruning for Reliability Evaluation Using Binary Particle Swarm Optimization,' in IEEE/PES Power Systems Conference and Exposition (PSCE), 2011, pp. 1-7.

[17] C. Singh and L. Wang, "Population-Based Intelligent Search in Reliability Evaluation of Generation Systems With Wind Power Penetration," IEEE Trans. Power Syst., vol. 23, no. 3, pp. 13361345, Aug. 2008.

[18] R. Green, L. Wang, Z. Wang, M. Alam, and C. Singh, "Power System Reliability Assessment Using Intelligent State Space Pruning Techniques : A Comparative Study," Int. Conf. Power Syst. Technol., pp. 1-8, 2010.

[19] Y. Valle, G. K. Venayagamoorthy, and R. G. Harley, "Particle Swarm Optimization : Basic Concepts, Variants and Applications in Power Systems," vol. 12, no. 2, pp. 171-195, 2008.

[20] R. a Bakkiyaraj and N. Kumarappan, "Evaluation of composite reliability indices based on non-sequential Monte Carlo simulation and particle swarm optimization," IEEE Congr. Evol. Comput., pp. 1-5, Jul. 2010.

[21] I. N. Kassabalidis, M. A. El-sharkawi, R. J. Marks, L. S. Moulin, and A. P. Alves, "Dynamic Security Border Identification Using Enhanced Particle Swarm Optimization," IEEE Trans. Power Syst., vol. 17 , no. 3, pp. 723-729, 2002.

[22] R. Green, L. Wang, and M. Alam, "Intelligent and Parallel State Space Pruning for Power System Reliability Analysis Using MPI on A Multicore Platform," in IEEE PES Innovative Smart Grid Technologies (ISGT), 2011, pp. 1-8.

[23] S. B. Patra, J. Mitra, and R. Earla, "A New Intelligent Search Method for Composite System Reliability Analysis," Transm. Distrib. Conf. Exhib. 2005/2006 IEEE PES, pp. 803 - 807.

[24] J. Mitra and X. Xu, "Composite System Reliability Analysis Using Particle Swarm Optimization," in IEEE 11th International Conference on Probabilistic Methods Applied to Power Systems (PMAPS), 2010, pp. 548-552.

[25] R. Green, L. Wang, and M. Alam, "Intelligent State Space Pruning Using Multi-Objective PSO for Reliability Analysis of Composite Power Systems : Observations , Analyses , and Impacts," in IEEE PES Innovative Smart Grid Technologies (ISGT), 2011, pp. 1-8.

[26] P. M. Subcommittee, "IEEE Reliability Test System," IEEE Trans. Power Appar. Syst., no. 6, pp. 2047-2054, 1979.

[27] R. D. Zimmerman, E. M.-S. Carlos, and D. Gan, "MATPOWER:A MATLAB Power System Simulation Package, Version 3.1b2, User's Manual.” Power Systems Engineering Research center(PSERC), Tech. Rep. 2006, New York, 2011.

[28] "http://badc.nerc.ac.uk/data/ukmo-midas/WPS.html."

[29] "IEEE Standard for Calculating the Current-Temperature of Bare Overhead Conductors," IEEE Std 738-2006 (Revision Std 7381993), pp. c1-59, 2007.

[30] K. Kopsidas, S. M. Rowland, and B. Boumecid, "A Holistic Method for Conductor Ampacity and Sag Computation on an OHL Structure," IEEE Trans. Power Deliv., vol. 27, no. 3, pp. 10471054, 2012.

[31] A. Kapetanaki, K. Kopsidas, C. Tumelo-Chakonta, and M. Buhari, "Network Planning Evaluation Implementing Time Varying Thermal Ratings," in PMAPS, 2014. 